\title{
A SHARP $L_{2}$ INEQUALITY OF OSTROWSKI TYPE
}

\author{
ZHENG LIU ${ }^{1}$
}

(Received 11 April, 2007)

\begin{abstract}
A sharp $L_{2}$ inequality of Ostrowski type is established, which provides a generalization of some previous results and gives some other interesting results as special cases. Applications in numerical integration are also given.

2000 Mathematics subject classification: 26D15.

Keywords and phrases: sharp bound, Ostrowski-type inequality, corrected trapezoid rule, corrected midpoint rule, corrected Simpson rule, corrected averaged midpointtrapezoid rule.
\end{abstract}

\section{Introduction}

In [1] and [2], we may find the following two interesting sharp bounds for the errors in the corrected trapezoid rule and corrected midpoint rule.

THEOREM 1.1. Let $f:[a, b] \rightarrow \mathbb{R}$ be such that $f^{\prime}$ is absolutely continuous on $[a, b]$ and $f^{\prime \prime} \in L_{2}[a, b]$. Then

$$
\begin{aligned}
& \left|\int_{a}^{b} f(t) d t-\frac{b-a}{2}[f(a)+f(b)]+\frac{(b-a)^{2}}{12}\left[f^{\prime}(b)-f^{\prime}(a)\right]\right| \\
& \quad \leq \frac{(b-a)^{(5 / 2)}}{12 \sqrt{5}} \sqrt{\sigma\left(f^{\prime \prime}\right)}
\end{aligned}
$$

where $\sigma(\cdot)$ is defined by

$$
\sigma(f)=\|f\|_{2}^{2}-\frac{1}{b-a}\left(\int_{a}^{b} f(t) d t\right)^{2}
$$

and $\|f\|_{2}:=\left[\int_{a}^{b} f^{2}(t) d t\right]^{(1 / 2)}$. Inequality (1.1) is sharp in the sense that the constant $(1 /(12 \sqrt{5}))$ cannot be replaced by a smaller one.

\footnotetext{
${ }^{1}$ Institute of Applied Mathematics, School of Science, University of Science and Technology Liaoning, Anshan 114051, Liaoning, China; e-mail: lewzheng@163.net.

(C) Australian Mathematical Society 2008, Serial-fee code 0334-2700/08
} 
THEOREM 1.2. Under the assumptions of Theorem 1.1,

$$
\begin{aligned}
& \left|\int_{a}^{b} f(t) d t-(b-a) f\left(\frac{a+b}{2}\right)-\frac{(b-a)^{2}}{24}\left[f^{\prime}(b)-f^{\prime}(a)\right]\right| \\
& \quad \leq \frac{(b-a)^{(5 / 2)}}{12 \sqrt{5}} \sqrt{\sigma\left(f^{\prime \prime}\right)} .
\end{aligned}
$$

Inequality (1.3) is sharp in the sense that the constant $1 / 12 \sqrt{5}$ cannot be replaced by a smaller one.

In this work, we will derive a new sharp inequality of Ostrowski type for functions whose first derivatives are absolutely continuous and whose second derivatives belong to $L_{2}(a, b)$. This will not only provide a generalization of inequalities (1.1) and (1.3), but will also give some other interesting sharp inequalities as special cases. Moreover, we show that the corrected Simpson rule (see [3-5]) gives better results than the Simpson rule and, in particular, the corrected averaged midpoint-trapezoid quadrature rule is optimal. Applications in numerical integration are also given.

\section{The results}

THEOREM 2.1. Let the assumptions of Theorem 1.1 hold. Then for any $\theta \in[0,1]$ and $x \in[a, b]$,

$$
\begin{aligned}
& \mid \int_{a}^{b} f(t) d t-(b-a)\left[(1-\theta) f(x)+\theta \frac{f(a)+f(b)}{2}\right] \\
& +(1-\theta)(b-a)\left(x-\frac{a+b}{2}\right) f^{\prime}(x) \\
& -\left[\frac{1-\theta}{2}\left(x-\frac{a+b}{2}\right)^{2}+\frac{1-3 \theta}{24}(b-a)^{2}\right]\left[f^{\prime}(b)-f^{\prime}(a)\right] \mid \\
& \leq\left[\frac{\theta(1-\theta)}{4}(b-a)\left(x-\frac{a+b}{2}\right)^{4}+\frac{3 \theta^{2}-5 \theta+2}{24}(b-a)^{3}\left(x-\frac{a+b}{2}\right)^{2}\right. \\
& \left.\quad+\frac{15 \theta^{2}-15 \theta+4}{2880}(b-a)^{5}\right]^{(1 / 2)} \sqrt{\sigma\left(f^{\prime \prime}\right) .}
\end{aligned}
$$

The inequality (2.1) is sharp in the sense that the coefficient constant 1 of the righthand side cannot be replaced by a smaller one.

PROOF. Let us define the function

$$
K(x, t):= \begin{cases}\frac{(t-a)^{2}}{2}-\frac{\theta(b-a)}{2}(t-a), & t \in[a, x], \\ \frac{(t-b)^{2}}{2}+\frac{\theta(b-a)}{2}(t-b), & t \in(x, b] .\end{cases}
$$


Integrating by parts, we obtain

$$
\begin{aligned}
\int_{a}^{b} K(x, t) f^{\prime \prime}(t) d t= & \int_{a}^{b} f(t) d t-(b-a)\left[(1-\theta) f(x)+\theta \frac{f(a)+f(b)}{2}\right] \\
& +(1-\theta)(b-a)\left(x-\frac{a+b}{2}\right) f^{\prime}(x) .
\end{aligned}
$$

We also have

$$
\int_{a}^{b} K(x, t) d t=\frac{1-\theta}{2}(b-a)\left(x-\frac{a+b}{2}\right)^{2}+\frac{1-3 \theta}{24}(b-a)^{3}
$$

and

$$
\int_{a}^{b} f^{\prime \prime}(t) d t=f^{\prime}(b)-f^{\prime}(a) .
$$

From (2.2)-(2.4), it follows that

$$
\begin{aligned}
\int_{a}^{b}\left[K(x, t)-\frac{1}{b-a} \int_{a}^{b} K(x, s) d s\right]\left[f^{\prime \prime}(t)-\frac{1}{b-a} \int_{a}^{b} f^{\prime \prime}(s) d s\right] d t \\
=\int_{a}^{b} f(t) d t-(b-a)\left[(1-\theta) f(x)+\theta \frac{f(a)+f(b)}{2}\right] \\
+(1-\theta)(b-a)\left(x-\frac{a+b}{2}\right) f^{\prime}(x) \\
\quad-\left[\frac{1-\theta}{2}\left(x-\frac{a+b}{2}\right)^{2}+\frac{1-3 \theta}{24}(b-a)^{2}\right]\left[f^{\prime}(b)-f^{\prime}(a)\right] .
\end{aligned}
$$

On the other hand,

$$
\begin{aligned}
\mid \int_{a}^{b} & {\left[K(x, t)-\frac{1}{b-a} \int_{a}^{b} K(x, s) d s\right]\left[f^{\prime \prime}(t)-\frac{1}{b-a} \int_{a}^{b} f^{\prime \prime}(s) d s\right] d t \mid } \\
& \leq\left\|K(x, \cdot)-\frac{1}{b-a} \int_{a}^{b} K(x, s) d s\right\|_{2}\left\|f^{\prime \prime}-\frac{1}{b-a} \int_{a}^{b} f^{\prime \prime}(s) d s\right\|_{2}(
\end{aligned}
$$

We also have

$$
\begin{aligned}
\left\|K(x, \cdot)-\frac{1}{b-a} \int_{a}^{b} K(x, s) d s\right\|_{2}^{2} \\
=\frac{\theta(1-\theta)}{4}(b-a)\left(x-\frac{a+b}{2}\right)^{4}+\frac{3 \theta^{2}-5 \theta+2}{24}(b-a)^{3}\left(x-\frac{a+b}{2}\right)^{2} \\
\quad+\frac{15 \theta^{2}-15 \theta+4}{4}(b-a)^{5}
\end{aligned}
$$


and

$$
\left\|f^{\prime \prime}-\frac{1}{b-a} \int_{a}^{b} f^{\prime \prime}(s) d s\right\|_{2}^{2}=\left\|f^{\prime \prime}\right\|_{2}^{2}-\frac{\left(f^{\prime}(b)-f^{\prime}(a)\right)^{2}}{b-a} .
$$

From (2.5)-(2.8), we can easily get (2.1), since by (1.2),

$$
\sqrt{\sigma\left(f^{\prime \prime}\right)}=\left[\left\|f^{\prime \prime}\right\|_{2}^{2}-\frac{\left(f^{\prime}(b)-f^{\prime}(a)\right)^{2}}{b-a}\right]^{(1 / 2)} .
$$

In order to prove that the inequality (2.1) is sharp, we define the function

$$
f(t)= \begin{cases}\frac{t^{4}}{24}-\frac{\theta t^{3}}{12}, & t \in[0, x], \\ \frac{(t-1)^{4}}{24}+\frac{\theta(t-1)^{3}}{12}+\left[\frac{1-\theta}{2}\left(x-\frac{1}{2}\right)^{2}+\frac{1-3 \theta}{24}\right] & \\ \quad \times\left(t-\frac{1}{2}\right)-\frac{1-\theta}{3}\left(x-\frac{1}{2}\right)^{3}, & t \in(x, 1],\end{cases}
$$

where $x \in[0,1]$. It follows that

$$
f^{\prime}(t)= \begin{cases}\frac{t^{3}}{6}-\frac{\theta t^{2}}{4}, & t \in[0, x], \\ \frac{(t-1)^{3}}{6}+\frac{\theta(t-1)^{2}}{4}+\frac{1-\theta}{2}\left(x-\frac{1}{2}\right)^{2}+\frac{1-3 \theta}{24}, & t \in(x, 1]\end{cases}
$$

and

$$
f^{\prime \prime}(t)= \begin{cases}\frac{t^{2}}{2}-\frac{\theta}{2} t, & t \in[0, x] \\ \frac{(t-1)^{2}}{2}+\frac{\theta}{2}(t-1), & t \in(x, 1]\end{cases}
$$

Clearly, the function given in (2.10) is absolutely continuous since it is a continuous piecewise polynomial function.

We now suppose that (2.1) holds with a constant $C>0$ as

$$
\begin{aligned}
& \mid \int_{a}^{b} f(t) d t-(b-a)\left[(1-\theta) f(x)+\theta \frac{f(a)+f(b)}{2}\right] \\
& +(1-\theta)(b-a)\left(x-\frac{a+b}{2}\right) f^{\prime}(x) \\
& \quad-\left[\frac{1-\theta}{2}\left(x-\frac{a+b}{2}\right)^{2}+\frac{1-3 \theta}{24}(b-a)^{2}\right]\left[f^{\prime}(b)-f^{\prime}(a)\right] \mid \\
& \quad \leq C\left[\frac{\theta(1-\theta)}{4}(b-a)\left(x-\frac{a+b}{2}\right)^{4}+\frac{3 \theta^{2}-5 \theta+2}{24}(b-a)^{3}\left(x-\frac{a+b}{2}\right)^{2}\right. \\
& \left.\quad+\frac{15 \theta^{2}-15 \theta+4}{2880}(b-a)^{5}\right]^{(1 / 2)} \sqrt{\sigma\left(f^{\prime \prime}\right) .}
\end{aligned}
$$


Choosing $a=0, b=1$, and $f$ defined in (2.9) with (2.10), (2.11), we get

$$
\begin{aligned}
\int_{0}^{1} f(t) d t= & \frac{1-\theta}{8}\left(x-\frac{1}{2}\right)^{4}-\frac{1-\theta}{6}\left(x-\frac{1}{2}\right)^{3}+\frac{1-\theta}{16}\left(x-\frac{1}{2}\right)^{2} \\
& +\frac{11-35 \theta}{1920}, \\
f(0)= & 0, \quad f(1)=\frac{1-\theta}{4}\left(x-\frac{1}{2}\right)^{2}-\frac{1-\theta}{3}\left(x-\frac{1}{2}\right)^{3}+\frac{1-3 \theta}{48} \\
f(x)= & \frac{1}{24}\left(x-\frac{1}{2}\right)^{4}+\frac{1-\theta}{12}\left(x-\frac{1}{2}\right)^{3}+\frac{1-2 \theta}{16}\left(x-\frac{1}{2}\right)^{2} \\
& +\frac{1-3 \theta}{48}\left(x-\frac{1}{2}\right)+\frac{1-4 \theta}{384}, \\
f^{\prime}(0)= & 0, \quad f^{\prime}(1)=\frac{1-\theta}{2}\left(x-\frac{1}{2}\right)^{2}+\frac{1-3 \theta}{24}, \\
f^{\prime}(x)= & \frac{1}{6}\left(x-\frac{1}{2}\right)^{3}+\frac{1-\theta}{4}\left(x-\frac{1}{2}\right)^{2}+\frac{1-2 \theta}{8}\left(x-\frac{1}{2}\right)+\frac{1-3 \theta}{48}
\end{aligned}
$$

and

$$
\int_{0}^{1}\left(f^{\prime \prime}(t)\right)^{2} d t=\frac{1-\theta}{4}\left(x-\frac{1}{2}\right)^{4}+\frac{2 \theta^{2}-3 \theta+1}{8}\left(x-\frac{1}{2}\right)^{2}+\frac{20 \theta^{2}-15 \theta+3}{960}
$$

such that the left-hand side becomes

$$
\text { L.H.S. (2.12) }=\frac{\theta(1-\theta)}{4}\left(x-\frac{1}{2}\right)^{4}+\frac{3 \theta^{2}-5 \theta+2}{24}\left(x-\frac{1}{2}\right)^{2}+\frac{15 \theta^{2}-15 \theta+4}{2880} \text {. }
$$

We also find that the right-hand side is

R.H.S. (2.12)

$$
=C\left[\frac{\theta(1-\theta)}{4}\left(x-\frac{1}{2}\right)^{4}+\frac{3 \theta^{2}-5 \theta+2}{24}\left(x-\frac{1}{2}\right)^{2}+\frac{15 \theta^{2}-15 \theta+4}{2880}\right] .
$$

From (2.12)-(2.14), we find that $C \geq 1$, proving that the coefficient constant 1 is the best possible in (2.1).

COROLlary 2.2. Let the assumptions of Theorem 2.1 hold. Then, for any $\theta \in[0,1]$,

$$
\begin{aligned}
& \mid \int_{a}^{b} f(t) d t-(b-a)\left[(1-\theta) f\left(\frac{a+b}{2}\right)+\theta \frac{f(a)+f(b)}{2}\right] \\
& -\frac{1-3 \theta}{24}(b-a)^{2}\left[f^{\prime}(b)-f^{\prime}(a)\right] \mid \\
& \quad \leq \frac{(b-a)^{(5 / 2)}}{24 \sqrt{5}}\left(15 \theta^{2}-15 \theta+4\right)^{(1 / 2)} \sqrt{\sigma\left(f^{\prime \prime}\right)} .
\end{aligned}
$$


Proof. We set $x=(a+b) / 2$ in (2.1) to get (2.15).

REMARK 1. If we take $\theta=1$ and $\theta=0$ in (2.15), then the sharp corrected trapezoid inequality (1.1) and the sharp corrected midpoint inequality (1.3) are recaptured. Thus Theorem 2.1 may be regarded as a generalization of Theorems 1.1 and 1.2.

REMARK 2. If we take $\theta=1 / 3$, we get a sharp Simpson-type inequality

$$
\left|\int_{a}^{b} f(t) d t-\frac{b-a}{6}\left[f(a)+4 f\left(\frac{a+b}{2}\right)+f(b)\right]\right| \leq \frac{(b-a)^{(5 / 2)}}{12 \sqrt{30}} \sqrt{\sigma\left(f^{\prime \prime}\right)} .
$$

If we take $\theta=7 / 15$, we get a sharp corrected Simpson-type inequality

$$
\begin{aligned}
& \left|\int_{a}^{b} f(t) d t-\frac{b-a}{30}\left[7 f(a)+16 f\left(\frac{a+b}{2}\right)+7 f(b)\right]+\frac{(b-a)^{2}}{60}\left[f^{\prime}(b)-f^{\prime}(a)\right]\right| \\
& \quad \leq \frac{(b-a)^{(5 / 2)}}{60 \sqrt{3}} \sqrt{\sigma\left(f^{\prime \prime}\right)} .
\end{aligned}
$$

From (2.16) and (2.17), we see that the corrected Simpson rule gives better results than the Simpson rule.

REMARK 3. If we take $\theta=1 / 2$, we get a sharp corrected averaged midpointtrapezoid-type inequality as

$$
\begin{aligned}
& \left|\int_{a}^{b} f(t) d t-\frac{b-a}{4}\left[f(a)+2 f\left(\frac{a+b}{2}\right)+f(b)\right]+\frac{(b-a)^{2}}{48}\left[f^{\prime}(b)-f^{\prime}(a)\right]\right| \\
& \quad \leq \frac{(b-a)^{(5 / 2)}}{48 \sqrt{5}} \sqrt{\sigma\left(f^{\prime \prime}\right)} .
\end{aligned}
$$

It is interesting to note that the smallest bound for (2.1) is obtained at $x=(a+b) / 2$ and $\theta=1 / 2$. Thus the corrected averaged midpoint-trapezoid rule is optimal in the current situation.

\section{Applications in numerical integration}

We restrict further considerations to the corrected averaged midpoint-trapezoid quadrature rule. We also emphasize that similar considerations can be given for all quadrature rules considered in the previous section.

THEOREM 3.1. Let $\pi=\left\{x_{0}=a<x_{1}<\cdots<x_{n}=b\right\}$ be a given subdivision of the interval $[a, b]$ such that $h_{i}=x_{i+1}-x_{i}=h=(b-a) / n$ and let the assumptions of Theorem 2.1 hold. Then

$$
\begin{aligned}
& \left|\int_{a}^{b} f(t) d t-\frac{h}{4} \sum_{i=0}^{n-1}\left[f\left(x_{i}\right)+2 f\left(\frac{x_{i}+x_{i+1}}{2}\right)+f\left(x_{i+1}\right)\right]+\frac{(b-a)^{2}}{48 n^{2}}\left[f^{\prime}(b)-f^{\prime}(a)\right]\right| \\
& \quad \leq \frac{(b-a)^{(5 / 2)}}{48 \sqrt{5} n^{2}} \sqrt{\sigma\left(f^{\prime \prime}\right)} .
\end{aligned}
$$


PROOF. From (2.18) we obtain

$$
\begin{aligned}
& \left|\int_{x_{i}}^{x_{i+1}} f(t) d t-\frac{h}{4}\left[f\left(x_{i}\right)+2 f\left(\frac{x_{i}+x_{i+1}}{2}\right)+f\left(x_{i+1}\right)\right]+\frac{h^{2}}{48}\left[f^{\prime}\left(x_{i+1}\right)-f^{\prime}\left(x_{i}\right)\right]\right| \\
& \quad \leq \frac{h^{(5 / 2)}}{48 \sqrt{5}}\left[\int_{x_{i}}^{x_{i+i}}\left(f^{\prime \prime}(t)\right)^{2} d t-\frac{1}{h}\left(f^{\prime}\left(x_{i+1}\right)-f^{\prime}\left(x_{i}\right)\right)^{2}\right]^{(1 / 2)}
\end{aligned}
$$

By summing (3.2) over $i$ from 0 to $n-1$ and using the generalized triangle inequality, we get

$$
\begin{aligned}
& \left|\int_{a}^{b} f(t) d t-\frac{h}{4} \sum_{i=0}^{n-1}\left[f\left(x_{i}\right)+2 f\left(\frac{x_{i}+x_{i+1}}{2}\right)+f\left(x_{i+1}\right)\right]+\frac{h^{2}}{48}\left[f^{\prime}(b)-f^{\prime}(a)\right]\right| \\
& \quad \leq \frac{h^{(5 / 2)}}{48 \sqrt{5}} \sum_{i=0}^{n-1}\left[\int_{x_{i}}^{x_{i+i}}\left(f^{\prime \prime}(t)\right)^{2} d t-\frac{1}{h}\left(f^{\prime}\left(x_{i+1}\right)-f^{\prime}\left(x_{i}\right)\right)^{2}\right]^{(1 / 2)} .
\end{aligned}
$$

By using the Cauchy inequality twice, it is not difficult to obtain

$$
\begin{aligned}
& \sum_{i=0}^{n-1}\left[\int_{x_{i}}^{x_{i+1}}\left(f^{\prime \prime}(t)\right)^{2} d t-\frac{1}{h}\left(f^{\prime}\left(x_{i+1}\right)-f^{\prime}\left(x_{i}\right)\right)^{2}\right]^{(1 / 2)} \\
& \quad \leq \sqrt{n}\left[\left\|f^{\prime \prime}\right\|_{2}^{2}-\frac{n}{b-a} \sum_{i=0}^{n-1}\left(f^{\prime}\left(x_{i+1}\right)-f^{\prime}\left(x_{i}\right)\right)^{2}\right]^{(1 / 2)} \\
& \quad \leq \sqrt{n}\left[\left\|f^{\prime \prime}\right\|_{2}^{2}-\frac{\left(f^{\prime}(b)-f^{\prime}(a)\right)^{2}}{b-a}\right]^{(1 / 2)}=\sqrt{n \sigma\left(f^{\prime \prime}\right)}
\end{aligned}
$$

Consequently, the inequality (3.1) follows from (3.3) and (3.4).

\section{References}

[1] N. B. Barnett, P. Cerone and S. S. Dragomir, "A sharp bound for the error in the corrected trapezoid rule and applications", Tamkang J. Math. 33 (2002) 253-258.

[2] P. Cerone, "On perturbed trapezoidal and midpoint rules", Korean J. Comput. Appl. Math. 2 (2002) 423-435.

[3] J. Pečaric and I. Franjić, "Generalization of corrected Simpson's formula", ANZIAM J. 47 (2006) $367-385$.

[4] N. Ujević, "A generalization of the modified Simpson's rule and error bounds", ANZIAM J. 47(E) (2005) E1-E13.

[5] N. Ujević and A. J. Roberts, "A corrected quadrature formula and applications", ANZIAM J. 45(E) (2004) E41-E56. 